\title{
Distributed causality in resting-state network connectivity in the acute and remitting phases of RRMS
}

Lin $\mathrm{Wu}^{1,2}$, Muhua Huang ${ }^{1,2}$, Fuqing Zhou ${ }^{1,2^{*}}$, Xianjun Zeng ${ }^{1,2}$ and Honghan Gong ${ }^{1,2}$

\begin{abstract}
Background: Although previous studies have shown that intra-network abnormalities in brain functional networks are correlated with clinical/cognitive impairment in multiple sclerosis (MS), there is little information regarding the pattern of causal interactions among cognition-related resting-state networks (RSNs) in different disease stages of relapsing-remitting MS (RRMS) patients. We hypothesized that abnormalities of causal interactions among RSNs occurred in RRMS patients in the acute and remitting phases.

Methods: Seventeen patients in the acute phases of RRMS, 24 patients in the remitting phases of RRMS, and 23 appropriately matched healthy controls participated in this study. First, we used group independent component analysis to extract the time courses of the spatially independent components from all the subjects. Then, the Granger causality analysis was used to investigate the causal relationships among RSNs in the spectral domain and to identify correlations with clinical indices.
\end{abstract}

Results: Compared with the patients in the acute phase of RRMS, patients in the remitting phase of RRMS showed a significantly lower expanded disability status scale, modified fatigue impact scale scores, and significantly higher paced auditory serial addition test (PASAT) scores. Compared with healthy subjects, during the acute phase, RRMS patients had significantly increased driving connectivity from the right executive control network (rECN) to the anterior salience network (aSN), and the causal coefficient was negatively correlated with the PASAT score. During the remitting phase, RRMS patients had significantly increased driving connectivity from the rECN to the aSN and from the rECN to the visuospatial network.

Conclusions: Together with the disease duration (mean disease duration $<5$ years) and relatively better clinical scores than those in the acute phase, abnormal connections, such as the information flow from the rECN to the aSN and the rECN to the visuospatial network, might provide adaptive compensation in the remitting phase of RRMS.

Keywords: Acute phase, Remitting phase, Multiple sclerosis, Resting-state networks, Granger causality

\section{Background}

Multiple sclerosis (MS) is a chronic inflammatory demyelination disease of the central nervous system that can cause progressive neurological deficits in young adults,

\footnotetext{
*Correspondence: fa.chou@yahoo.com

1 Department of Radiology, The First Affiliated Hospital, Nanchang

University, Nanchang, Jiangxi, People's Republic of China

Full list of author information is available at the end of the article
}

including up to $70 \%$ cognitive impairment [1]. Developments in functional magnetic resonance imaging (fMRI) could help to further unravel the underlying mechanisms that cause these cognitive deficits [2]. Notably, fMRI data have been used to examine the functional connectivity (FC) of spatially remote brain regions to delineate a set of functional networks that exhibit high reproducibility and moderate to high test-retest reliability [3-5]. Some studies in MS have found that the default mode network 
$(\mathrm{DMN})$, which is associated with internally directed cognition, exhibits decreased activation during cognitive task performance [6] and disturbed FC within DMN regions during rest [7-9]. Additionally, patients with MS with cognitive impairment display reduced eigenvector centrality dynamics in the DMN, frontoparietal network, and visual network [10]. Our previous study found that abnormalities of the attention network, which is more associated with externally directed cognition, have been observed in relapsing-remitting MS (RRMS); these abnormalities include decreased FC [11] and dynamic FC [12] within the network. Overall, previous studies have demonstrated that extensive functional networks present dysfunction, which is related to the cognitive performance in MS patients.

To better understand the cognitive functions of the human brain, it is essential not only to study individual or intrinsic functional networks but also to consider how they work together as a whole. A functional network connectivity study found that abnormalities of functional interactions between the principal resting-state networks (RSNs) were also present in RRMS patients and were related to the severity of the disability [13]. Recently, a few studies have shown abnormal functional connection strength among the DMN, attention network and working memory network in paediatric MS [14] and the maintenance of a stable interaction between the DMN and attention network in adult RRMS patients in the remitting phase [11]. Moreover, previous MS studies indicated that structural brain damage might result in functional reorganization (increased FC) before the functional network collapses, prompting a delayed cognitive decline [15]. However, either an increase or decrease in the FC of the internetwork correlates with a decline in cognition [9, 15-17]. In truth, we are currently unable to simplistically think whether the increased functional connection is good or bad. Additionally, previous studies did not account for causal interactions among cognitionrelated RSNs in different stages of disease in patients with RRMS. Granger causality analysis (GCA) provides a powerful and generic statistical tool for characterizing directed functional interactions from time-series data. Yan et al. [18] found the GCA-based functional directed network of the human brain is the stability and reproducibility and suggested the GCA might be a reliable approach for the performance of a spontaneous causal influence analysis with resting-state fMRI.

Therefore, we hypothesized that abnormalities in the causal interactions among RSNs occurred in RRMS patients in the acute and remitting phases. We first used group independent component analysis (ICA) to extract RSN time courses in patients with RRMS and healthy controls and identified 14 consistent large-scale RSNs through a voxelwise correlation with a template-matching algorithm [19]. Then, to quantify the strength of interactions and to reveal directed interactions between independent components, the GCA was used to investigate interactions among fourteen RSNs. Connectivity was compared between patients experiencing the acute phase of RRMS and healthy controls and between patients experiencing the remitting phase of RRMS and healthy controls. Subsequently, we assessed the correlation between altered causal coefficients and the clinical profile in RRMS. This study may help our understanding of the correlation between brain functional network rewiring and clinical dysfunction in RRMS.

\section{Materials and methods \\ Subjects}

From May 2014 to December 2018, a total of 41 patients with clinically diagnosed RRMS (17 in acute phases and 24 in remitting phases) were recruited at the First Affiliated Hospital of Nanchang University, according to the 2010 revised McDonald's criteria [20]. Twenty-three appropriately matched (age, sex, and education) subjects served as healthy controls ( $\mathrm{HCs}$ ). The exclusion criteria for the subjects were the presence or history of traumatic brain injuries, tumour or stroke based on conventional MRI data. All subject have self-reported being right-handed.

All the patients underwent neuropsychological evaluations, including the Expanded Disability Status Scale (EDSS), Modified Fatigue Impact Scale (MFIS), and Paced Auditory Serial Addition Test (PASAT).

\section{Image acquisition and preprocessing}

All the subjects were imaged with a 3.0 T MRI scanner (Trio Tim; Siemens, Munich, Germany) using an eightchannel phased array head coil. The following sequences of the brain were acquired: (1) T2\%-weighted gradient echo sequence $\left(\mathrm{TR} / \mathrm{TE}=2,000 / 30 \mathrm{~ms}\right.$, flip angle $=90^{\circ}$, $\mathrm{FOV}=200 \times 200 \mathrm{~mm}$, matrix $=64 \times 64,30$ interleaved axial slices with a $4 \mathrm{~mm}$ thickness and an interslice gap of $1.2 \mathrm{~mm}$, number of time points $=240$ ); (2) T2-weighted turbo spin-echo imaging; and (3) three-dimensional T1-weighted imaging. During the RS-fMRI scanning, the subjects were instructed simply to rest with their eyes closed, not to think systematically, and not to fall asleep.

All the preprocessing steps were carried out using the MATLAB 2012a platform (MathWorks, Inc., Natick, MA, USA). The standard preprocessing procedures have been described in our previous studies [11]. The main steps of preprocessing included the first ten volumes of each session were discarded for the equilibrium state of the echo signal, slice correction, head realignment, spatial normalization to MNI space with high-resolution 
T1WI registration, resampling to $3 \mathrm{~mm}$ isotropic voxels, and $6 \mathrm{~mm}$ smoothing. Besides, subjects with head movement in the cardinal directions $(x, y, z)>2 \mathrm{~mm}$ and a maximum rotation $(x, y, z)>2^{\circ}$ were excluded.

\section{Group ICA}

We performed a group spatial ICA on the preprocessed data of the patients with RRMS and normal controls using the Group ICA of fMRI Toolbox (GIFT, https:// icatb.sourceforge.net/groupica.htm). We chose a relatively high model order ICA (number of components, $\mathrm{C}=75$ ), as previous studies have demonstrated that such models yield refined components [21] and a highly stable ICA decomposition [22]. In the group ICA, the mean independent components of all the subjects, the corresponding mean time courses and the independent components for each subject were obtained from the group ICA separation and back reconstruction to ensure that all the subjects had the same components [23]. After standard preprocessing of the group ICA results, from 75 components, we identified fourteen RSNs via a templatematching algorithm based on the maximum spatial correlation value. These functional templates were provided by Shirer et al. [19]. Then, one-sample t-test of the groupwise spatial maps was performed by Data Processing Assistant for Resting-State fMRI Advanced Edition (version 2.2).

\section{GCA}

The GCA in a spectral method that is used to elucidate the causal relationships between two or more stationary variables for brain networks [24]. Based on the principle of Granger causality, if incorporating the past values of time series $X$ improves the future prediction of time series $\mathrm{Y}$, then $\mathrm{X}$ is said to have a causal influence on $\mathrm{Y}$ [25]. Granger causality analysis was accomplished using the functional network connectivity toolbox (https:// icatb.sourceforge.net/). According to a given interval and an order selection criterion, the optimal order of the autoregressive model was selected, which was not constant and varied within the interval for every mutual relationship. We preferred the Schwartz Bayesian criterion to determine the optimal order of the autoregressive model and to obtain the smallest mean-squared prediction error of the fitted autoregressive model. In this study, the GCA was compared between the acute phase of RRMS and healthy controls and between the remitting phase of RRMS and healthy controls. The statistical significance level was set at a $P$-value less than 0.05 with false discovery rate (FDR) correction.

\section{Statistical analysis}

We performed statistical analyses of the demographic, clinical, and relationship data by using SPSS software (version 22, 2013; IBM, Chicago, Ill). We investigated Pearson's correlation between abnormal Granger causality coefficients and neuropsychological characteristics in RRMS while controlling for age and sex. The relationship was significant if the $P$-value was below 0.05 .

\section{Results \\ Demographic and clinical data}

Table 1 shows the demographic and clinical characteristics of the RRMS patients and HCs. Significant differences in the EDSS, PASAT and MFIS were observed between the acute phase and remitting phase of RRMS.

\section{Spatial distribution of RSNs}

An analysis of the fMRI data revealed 14 spatial maps of potentially relevant RSNs, including the posterior salience network (IC 8), anterior salience network (aSN) (IC 10), basal ganglia network (IC 20), primary visual

Table 1 Demographic and clinical characteristics of the study population

\begin{tabular}{|c|c|c|c|c|c|c|}
\hline & \multicolumn{2}{|l|}{ RRMS } & \multirow[t]{2}{*}{$H C s(n=23)$} & \multirow[t]{2}{*}{$P$-values ${ }^{\mathrm{a}}$} & \multirow[t]{2}{*}{$P$-values ${ }^{b}$} & \multirow[t]{2}{*}{$P$-values ${ }^{c}$} \\
\hline & Acute phase $(n=17)$ & $\begin{array}{l}\text { Remitting phase } \\
(n=24)\end{array}$ & & & & \\
\hline Gender (M/F) & $7 / 10$ & $9 / 15$ & $12 / 11$ & 0.504 & 0.322 & $\mathrm{n} / \mathrm{a}$ \\
\hline Mean age (range) (years) & $43.1(15-61)$ & $40.7(21-66)$ & $40.7(26-58)$ & 0.446 & 0.996 & $\mathrm{n} / \mathrm{a}$ \\
\hline $\begin{array}{l}\text { Mean disease duration (range) } \\
\text { (months) }\end{array}$ & $23.3(2-55)$ & $31.1(4-150)$ & - & $\mathrm{n} / \mathrm{a}$ & $\mathrm{n} / \mathrm{a}$ & $\mathrm{n} / \mathrm{a}$ \\
\hline Mean EDSS (range) & $2.8(1.5-4)$ & $2.0(0-3.5)$ & - & $\mathrm{n} / \mathrm{a}$ & $\mathrm{n} / \mathrm{a}$ & 0.015 \\
\hline Mean PASAT (range) & $88.3(76-103)$ & $90.2(80-103)$ & - & $\mathrm{n} / \mathrm{a}$ & $\mathrm{n} / \mathrm{a}$ & 0.002 \\
\hline Mean MFIS (range) & $11.2(6-16)$ & $9.0(2-15)$ & - & $\mathrm{n} / \mathrm{a}$ & $\mathrm{n} / \mathrm{a}$ & 0.03 \\
\hline
\end{tabular}

\footnotetext{
a Comparison between the acute phase of RRMS and healthy controls

b Comparison between the remitting phase of RRMS and healthy controls

c Comparison between the acute phase and remitting phase of RRMS
} 
network (IC 23), visuospatial network (IC 28), higher visual network (IC 58), language network (IC 59), sensorimotor network (IC 62), auditory network (IC 63), left executive control network (IC 66), right executive control network (rECN) (IC 68), dorsal DMN (IC 70), praecuneus network (IC 74), and ventral DMN (IC 75). (Fig. 1 and Additional file 1: Fig. S1).

\section{Causal relationships of RSNs}

We also used the GCA to investigate the causal relationships of fourteen RSNs compared between the RRMS and HCs. During the acute phase of RRMS, the patients had a significantly increased driving connectivity from the rECN to the aSN. (Fig. 2 and Additional file 1: Fig. S2). During the remitting phase of RRMS, the patients had significantly increased driving connectivity from the rECN to the aSN and from the rECN to the visuospatial network. (Fig. 3 and Additional file 1: Fig. S3) $(\mathrm{P}<0.05$, FDR correction).

\section{Analysis of correlations}

Our study only observed that an increased causal coefficient from the rECN to the aSN was negatively correlated with the PASAT score in the acute phase of RRMS $(r=-0.528, P=0.029)$. The other abnormal causal coefficients did not show significant correlations with the clinical parameters (including disease duration, EDSS, PASAT and MFIS) (Fig. 4).

\section{Discussion}

We applied group ICA and Granger causality to reveal relationships among fMRI brain networks in the spectral domain between RRMS patients and HCs. Depending on the different stages of the disease, patients with RRMS were observed to have different network causal connections. These results represent the general direction of the information flow. This approach investigating the direction of causality among cognition-related RSNs can form a useful complement to aid in understanding the underlying compensatory mechanism in MS.

\section{Comparison between patients in the acute phase of RRMS and HCs}

ECN and aSN dysfunctions have been described in MS $[13,26]$. The ECN is functionally related to but dimensionally separate from the aSN. While the ECN is thought to be involved in goal-directed behavior and perceptual processes [27], the primary roles of the aSN are associated with stimulus-driven processes, detecting salient cues in the environment, interrupting ongoing activity in the ECN [28] and coordinating neural resources [29, 30]. The FC between these two networks has been described to guide interactions with the external environment and responses to potentially threatening stimuli [31]. Moreover, a study of the Stroop interference task showed that the rECN had the greatest number of outgoing connections and sent information to the insula (the key nodes of the $\mathrm{SN}$ ), and the study noted that the abnormal interaction of MS patients might correlate with deficits of cognitive performance [32]. Our results represent the general direction of the information flow from the rECN to the aSN in the acute phase of RRMS in task-free conditions. Interestingly, we found that an increased causal coefficient was negatively correlated with the PASAT score. Historically, most early studies have used the PASAT to study cognition in MS, as it measures sustained attention and information processing speed [33, 34]. Although this neural mechanism facilitates access to resources that engage the PASAT task by activating the $\operatorname{rECN}[6,35]$, one should exercise caution regarding the interpretation

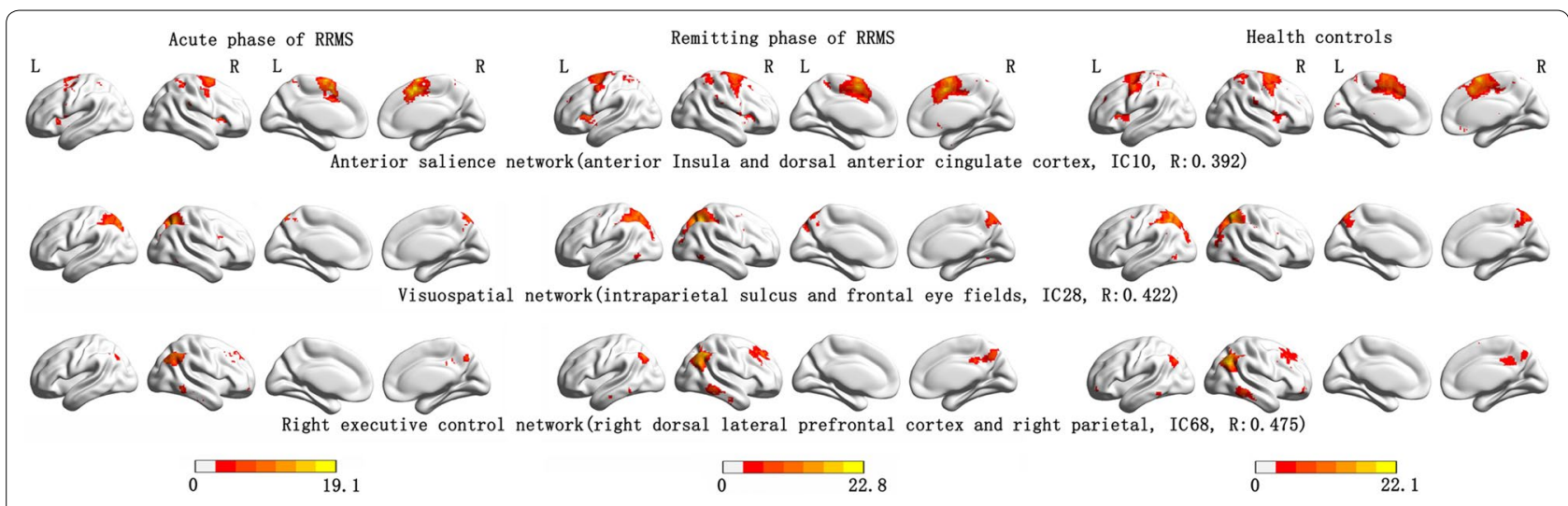

Fig. 1 Spatial map of the three resting state networks from relapsing-remitting multiple sclerosis and health controls. (one-sample $t$-test, $P=0.001$, FDR correction). IC 10: independent component 10 obtained by group independent components analysis. $\mathrm{R}$ is the highest correlation coefficient which results from the spatial match of the independent components with the offering templates 


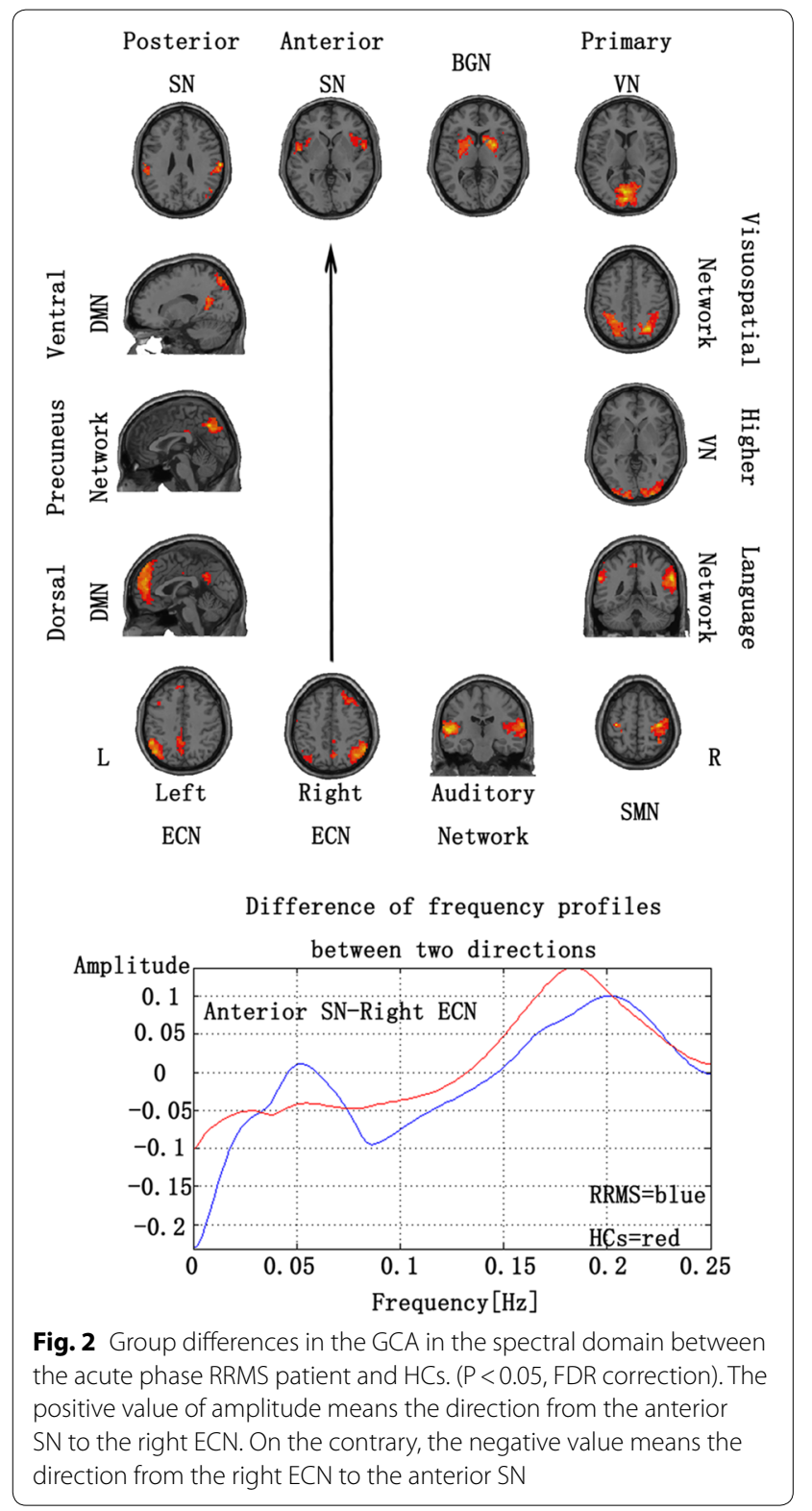

of how causal changes may underlie clinical and cognitive deterioration in MS. Future longitudinal assessments will provide the opportunity to define whether an abnormal causal relationship has a role in the changes in cognitive function in the acute phase of RRMS.

\section{Comparison between patients in the remitting phase of RRMS and HCs}

Compared with HCs, we also observed significantly increased driving connectivity from the rECN to the aSN and the visuospatial network in patients in the remitting phase of RRMS. Our partial result is consistent with a previous functional network connectivity study in which the ECN had increased connectivity with the $\mathrm{SN}$ in the remitting phase of RRMS in task-free conditions [13]. However, no previous studies have reported driving connectivity from the rECN to the visuospatial network in the remitting phase of RRMS. The visuospatial network, whose core regions include the intraparietal sulcus and the frontal eye field, is related to a specific modulation of corresponding locations of visual perception [36]. The visuospatial network regions send top-down biases to visual areas via the middle frontal gyrus (a core region of the ECN) to the ventral attention system, the core regions of which include the anterior insula, restricting ventral activation to behaviorally important stimuli. Conversely, when attention is reoriented to a new source of information (stimulus-driven reorienting), the ventral attention system sends a reorienting signal to the visuospatial network through the middle frontal gyrus (as a circuit breaker), which shifts attention towards the novel object of interest [37, 38]. In summary, neuroimaging evidence suggests that when novel stimuli involving cognition and emotion are present, the interaction among the visuospatial network, the ECN and the SN would form the control systems of the brain to respond stimuli [31, 39].

Recent studies also show that shorter and longer RRMS disease duration with similar disabilities are characterized by distinct patterns of FC, involving predominantly sensory and cognitive networks, respectively [40]. Besides, increased FC between brain regions implies the use of brain reserves to ameliorate cognitive impairment in early-stage MS patients (disease duration $<5$ years), which is generally interpreted as a compensatory mechanism [34, 41]. During the course of disease after reaching a maximal level, brain FC enhancement decreases and decreased FC participates in disability progression, according to a longitudinal resting-state fMRI study [42]. Moreover, an anatomo-functional study revealed that a brain network disconnection may deprive the brain of compensatory mechanisms in cognitively preserved patients with early MS (3-5 years disease duration) [8]. Taken together, these studies suggest that in patients with shorter disease duration (mean disease duration $<5$ years) and relatively better clinical scores (EDSS, PASAT, MFIS) in the remitting phase compared to those in the acute phase of RRMS, increased driving connectivity from the rECN to the aSN and from the rECN to the visuospatial network may be an adaptive compensatory mechanism to limit the clinical consequences of diseaserelated tissue damage.

In the end, our study shows opposite directions of causal association between low and high frequency ranges. For example, in Fig. 2, the high frequency range is indicated as the direction from the aSN to the rECN. On the contrary, low frequency range means the direction 

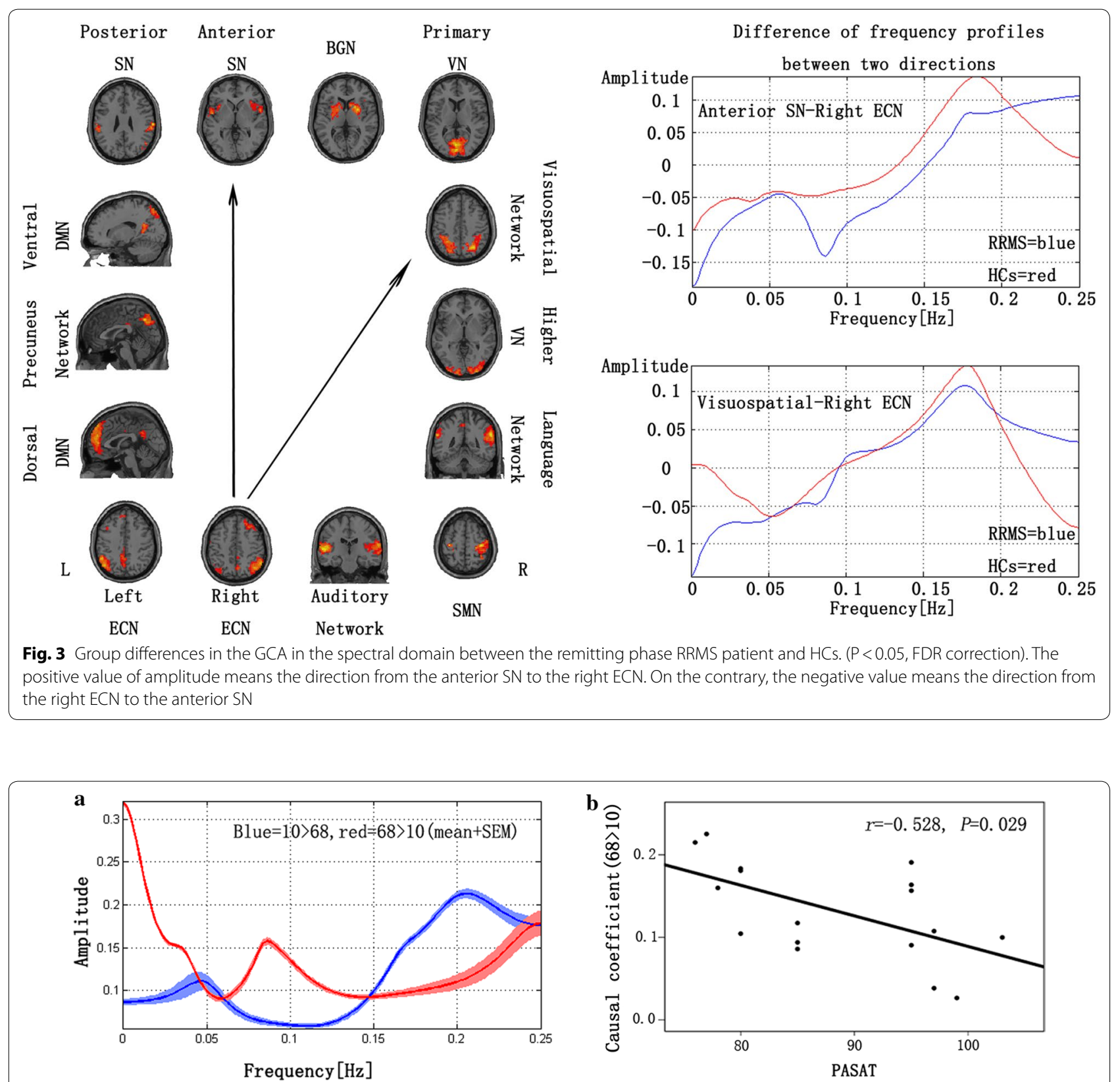

Fig. 4 Correlations between the PASAT score and the altered connectivity coefficients in the acute phase of RRMS. a Frequency profiles for both directions; b Pearson's correlation scatter plot. 68> 10 means causal coefficient from component 68 (right executive control network) to component 10 (anterior salience network)

from the rECN to the aSN. Directional relationships in low and high frequencies could implicate different causal associations. Causality in a low frequency could suggest that a sustained state was alternated to the other state in the identified order. In contrast, causality in a high frequency could suggest a transfer of frequent transient activation between the networks. Although the order of activations in a short interval is hard to identify due to a short TR (2-s) and variability of the hemodynamic response function, a slow network alteration in low-frequency range can be validated. The part of low-frequency caused the difference between the groups in our study (showing Additional file 1: Figs. S2, S3). Future researches about Granger prediction between different frequency bands will give us more useful insights into the pathology of RRMS. 


\section{Limitations}

First, altered FC is related to the level of structural damage and distribution of MS lesions [9, 12], and it has also been characterized by mostly heterogeneous findings. For instance, the consensus is that MS lesions disrupt the corresponding white matter pathways, leading to dysfunction. However, recent research has suggested that physical and cognitive disabilities seem to correlate better with damage to grey matter than with the lesion load of white matter in MS patients [43]. Future research is needed to elucidate the association between structural damage (grey matter and white matter) and causal changes involving cortical and subcortical RSNs in MS. Second, it should be noted that the observed correlation in the acute phase was relatively weak, and no correlations were observed in the remitting phase of RRMS. Undeniably, our acquired images $(4 \mathrm{~mm}$ slice thickness and 2-s TR) limited the spatial and time resolution research on this "causality" to a certain extent. Thus, higher-resolution fMRI data (especially with shorter TR scanning) studies should be conducted in the future. Third, the result of correlation analysis $(\mathrm{P}=0.029)$ cannot survive the correction for testing multiple scales. Therefore, a greater number of subjects should be used in future studies.

\section{Conclusion}

In summary, we found abnormalities in causal interactions between cognition-related RSNs in patients in the acute phases and remitting phases of RRMS compared with HCs. These findings, together with the disease duration and relatively better clinical scores in patients in the remitting phase than in the acute phase, highlight the complexity of the interactions between RSNs that might act as adaptive compensatory mechanisms in the remitting phase of RRMS. A longitudinal study in the future may help to determine the extent of connectivity changes between the resting state networks with the progression of the disease.

\section{Supplementary information}

Supplementary information accompanies this paper at https://doi. org/10.1186/s12868-020-00590-4.

Additional file1 (DOCX 1556 kb)

\section{Abbreviations}

MS: multiple sclerosis; fMRl: functional magnetic resonance imaging; FC: functional connectivity; DMN: default mode network; RRMS: relapsing-remitting MS; RSNs: resting-state networks; ICA: independent component analysis; GCA : granger causality analysis; HCs: healthy controls; EDSS: disability status scale; MFIS: modified fatigue impact scale; PASAT: paced auditory serial addition test; TR: repetition time; TE: echo time; FDR: false discovery rate; aSN: anterior salience network; BGN: basal ganglia network; rECN: right executive control network; VN: visual network; SEM: standard error of mean.

\section{Acknowledgements}

The authors thank their patients and volunteers for participating in this study.

\section{Authors' contributions}

LW and FZ conceived and designed the study, XZ and HG supervised the research. LW and $\mathrm{MH}$ collected and analyzed data. LW and FZ interpreted data. LW have drafted the work. HG substantively revised it. All authors read and approved the final manuscript.

\section{Funding}

This study was supported by the National Science Foundation of China (Grant Nos. 81560284 and 81771808), the Natural Science Foundation of Jiangxi, China (Grant No. 2017BAB215048), the Science and Technology Project of Jiangxi Health Committee (Grant No. 20181020), the Science and Technology Research Project of Jiangxi Provincial Department of Education (Grant No. 700993003)

\section{Availability of data and materials}

All raw data are stored in Department of Radiology, The First Affiliated Hospital, Nanchang University. The datasets analyses are available from the corresponding author on reasonable request.

\section{Ethics approval and consent to participate}

This study was approved by the Medical Research Ethics Committee and the Institutional Review Board of the First Affiliated Hospital of Nanchang University. All participants provided their written informed consent to participate. In participants with cognitive decline, informed consent is obtained from a legal guardian or representative because they were not capable of providing ethical consent by themselves.

Consent for publication

Not applicable.

\section{Competing interests}

The authors declare that they have no competing interests.

\section{Author details}

${ }^{1}$ Department of Radiology, The First Affiliated Hospital, Nanchang University, Nanchang, Jiangxi, People's Republic of China. ${ }^{2}$ Jiangxi Province Medical Imaging Research Institute, Nanchang, Jiangxi, People's Republic of China.

Received: 18 March 2020 Accepted: 9 September 2020

Published online: 15 September 2020

\section{References}

1. Chiaravalloti ND, DeLuca J. Cognitive impairment in multiple sclerosis. Lancet Neurol. 2008;7:1139-51.

2. Benedict $\mathrm{RH}$, Zivadinov R. Risk factors for and management of cognitive dysfunction in multiple sclerosis. Nat Rev Neurol. 2011;7:332-42.

3. Jafri MJ, Pearlson GD, Stevens M, Calhoun VD. A method for functional network connectivity among spatially independent resting-state components in schizophrenia. Neuroimage. 2008;39:1666-811.

4. Smith SM, Fox PT, Miller KL, Glahn DC, Fox PM, Mackay CE, et al. Correspondence of the brain's functional architecture during activation and rest. Proc Natl Acad Sci USA. 2009;106:13040-5.

5. Zuo XN, Kelly C, Adelstein JS, Klein DF, Castellanos FX, Milham MP. Reliable intrinsic connectivity networks: test-retest evaluation using ICA and dual regression approach. Neuroimage. 2010;49:2163-77.

6. Rocca MA, Valsasina P, Hulst HE, Abdel-Aziz K, Enzinger C, Gallo A, et al. Functional correlates of cognitive dysfunction in multiple sclerosis: a multicenter fMRI Study. Hum Brain Mapp. 2014;35:5799-814.

7. Greicius MD, Krasnow B, Reiss AL, Menon V. Functional connectivity in the resting brain: a network analysis of the default mode hypothesis. Proc Natl Acad Sci USA. 2003;100:253-8. 
8. Louapre C, Perlbarg V, Garcia-Lorenzo D, Urbanski M, Benali H, Assouad R, et al. Brain networks disconnection in early multiple sclerosis cognitive deficits: an anatomofunctional study. Hum Brain Mapp. 2014;35:4706-17.

9. Rocca MA, Valsasina P, Leavitt VM, Rodegher M, Radaelli M, Riccitelli GC, et al. Functional network connectivity abnormalities in multiple sclerosis: correlations with disability and cognitive impairment. Mult Scler. 2018;24:459-71.

10. Eijlers AJC, Wink AM, Meijer KA, Douw L, Geurts JJG, Schoonheim MM. Reduced network dynamics on functional MRI signals cognitive impairment in multiple sclerosis. Radiology. 2019;292:449-57.

11. Huang M, Zhou F, Wu L, Wang B, Wan H, Li F, et al. Synchronization within, and interactions between, the default mode and dorsal attention networks in relapsing-remitting multiple sclerosis. Neuropsychiatr Dis Treat. 2018;14:1241-52.

12. Huang M, Zhou F, Wu L, Wang B, Guo L, Zhao Y, et al. White matter lesion loads associated with dynamic functional connectivity within attention network in patients with relapsing-remitting multiple sclerosis. J Clin Neurosci. 2019;65:59-655.

13. Rocca MA, Valsasina P, Martinelli V, Misci P, Falini A, Comi G, et al. Largescale neuronal network dysfunction in relapsing-remitting multiple sclerosis. Neurology. 2012;79:1449-577.

14. Rocca MA, Valsasina P, Absinta M, Moiola L, Ghezzi A, Veggiotti P, et al. Intranetwork and internetwork functional connectivity abnormalities in pediatric multiple sclerosis. Hum Brain Mapp. 2014;35:4180-92.

15. Schoonheim MM, Meijer KA, Geurts JJ. Network collapse and cognitive impairment in multiple sclerosis. Front Neurol. 2015;6:82.

16. Pantano P, Mainero C, Caramia F. Functional brain reorganization in multiple sclerosis: evidence from fMRI studies. J Neuroimaging. 2006;16:104-14

17. Chirumamilla VC, Fleischer V, Droby A, Anjum T, Muthuraman M, Zipp $F$, et al. Functional connectivity analysis using whole brain and regional network metrics in MS patients. Conf Proc IEEE Eng Med Biol Soc. 2016;2016:4039-42.

18. Yan CG, He Y. Driving and driven architectures of directed small-world human brain functional networks. PLoS ONE. 2011;6:e23460.

19. Shirer WR, Ryali S, Rykhlevskaia E, Menon V, Greicius MD. Decoding subject-driven cognitive states with whole-brain connectivity patterns. Cereb Cortex. 2012;22:158-65.

20. Polman CH, Reingold SC, Banwell B, Clanet M, Cohen JA, Filippi M, et al. Diagnostic criteria for multiple sclerosis: 2010 revisions to the McDonald criteria. Ann Neurol. 2011;69:292-302.

21. Ystad M, Eichele T, Lundervold AJ, Lundervold A. Subcortical functional connectivity and verbal episodic memory in healthy elderly-a resting state fMRI study. Neuroimage. 2010;52:379-88.

22. Allen EA, Erhardt EB, Damaraju E, Gruner W, Segall JM, Silva RF, et al. A baseline for the multivariate comparison of resting-state networks. Front Syst Neurosci. 2011;5:2.

23. Calhoun VD, Adali T, Pearlson GD, Pekar JJ. A method for making group inferences from functional MRI data using independent component analysis. Hum Brain Mapp. 2001;14:140-51.

24. Demirci O, Stevens M, Andreasen NC, Michael A, Liu J, White T, Pearlson GD, Clark VP, Calhoun VD, et al. Investigation of relationships between fMRI brain networks in the spectral domain using ICA and Granger causality reveals distinct differences between schizophrenia patients and healthy controls. Neuroimage. 2009;46:419-31.

25. Granger CWJ. Investigating causal relations by econometric models and cross-spectral methods. Econometrica. 1969;37:424-38.

26. Benedict RH, Zivadinov R. Default mode network changes in multiple sclerosis: a link between depression and cognitive impairment? Eur J Neurol. 2017;24:27-36.
27. Seeley WW, Menon V, Schatzberg AF, Keller J, Glover GH, Kenna H, et al. Dissociable intrinsic connectivity networks for salience processing and executive control. J Neurosci. 2007;27:2349-56.

28. Corbetta M, Patel G, Shulman GL. The reorienting system of the human brain: from environment to theory of mind. Neuron. 2008;58:306-24.

29. Sridharan D, Levitin DJ, Menon V. A critical role for the right fronto-insular cortex in switching between central-executive and default-mode networks. Proc Natl Acad Sci USA. 2008;105:12569-74.

30. Uddin LQ. Salience processing and insular cortical function and dysfunction. Nat Rev Neurosci. 2015;16:55-61.

31. Provenzano J, Verduyn P, Daniels N, Fossati P, Kuppens P. Mood congruency effects are mediated by shifts in salience and central executive network efficiency. Soc Cogn Affect Neurosci. 2019;14:987-95.

32. Dobryakova E, Rocca MA, Valsasina P. Abnormalities of the executive control network in multiple sclerosis phenotypes: an fMRI effective connectivity study. Hum Brain Mapp. 2016;37:2293-304.

33. Staffen W, Mair A, Zauner H, Unterrainer J, Niederhofer H, Kutzelnigg A, et al. Cognitive function and fMRI in patients with multiple sclerosis: evidence for compensatory cortical activation during an attention task. Brain. 2002;125:1275-82.

34. Audoin B, Ibarrola D, Ranjeva JP, Confort-Gouny S, Malikova I, Ali-Chérif A, et al. Compensatory cortical activation observed by fMRI during a cognitive task at the earliest stage of MS. Hum Brain Mapp. 2003;20:51-8.

35. Mainero C, Caramia F, Pozzilli C, Pisani A, Pestalozza I, Borriello G, et al. fMRI evidence of brain reorganization during attention and memory tasks in multiple sclerosis. Neuroimage. 2004;21:858-67.

36. Ruff CC, Bestmann S, Blankenburg F, Bjoertomt O, Josephs O, Weiskopf $\mathrm{N}$, et al. Distinct causal influences of parietal versus frontal areas on human visual cortex: evidence from concurrent TMS-fMRI. Cereb Cortex. 2008; 18:817-27.

37. Corbetta M, Shulman GL. Control of goal-directed and stimulus-driven attention in the brain. Nat Rev Neurosci. 2002;3:201-15.

38. Hopfinger JB, Buonocore MH, Mangun GR. The neural mechanisms of top-down attentional control. Nat Neurosci. 2000;3:284-91.

39. Dosenbach NU, Fair DA, Cohen AL, Schlaggar BL, Petersen SE. A dual-networks architecture of top-down control. Trends Cogn Sci. 2008;12:99-105.

40. Castellazzi G, Debernard L, Melzer TR, Dalrymple-Alford JC, D'Angelo E, Miller DH, et al. Functional connectivity alterations reveal complex mechanisms based on clinical and radiological status in mild relapsing remitting multiple sclerosis. Front Neurol. 2018;9:690.

41. Lopez-Gongora M, Escartin A, Martinez-Horta S, Dalrymple-Alford JC, D'Angelo E, Miller DH, et al. Neurophysiological evidence of compensatory brain mechanisms in early-stage multiple sclerosis. PLOS ONE. 2015;10:e0136786.

42. Faivre A, Robinet E, Guye M, Rousseau C, Maarouf A, Le Troter A, et al. Depletion of brain functional connectivity enhancement leads to disability progression in multiple sclerosis: a longitudinal resting-state fMRI study. Mult Scler. 2016;22:1695-708.

43. Calabrese M, Reynolds R, Magliozzi R, Castellaro M, Morra A, Scalfari $A$, et al. Regional distribution and evolution of gray matter damage in different populations of multiple sclerosis patients. PLOS ONE. 2015;10:e0135428.

\section{Publisher's Note}

Springer Nature remains neutral with regard to jurisdictional claims in published maps and institutional affiliations. 